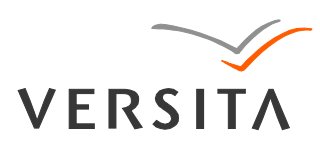

GEOCHRONOMETRIA 41(2) 2014: 160-167

DOI 10.2478/s13386-013-0147-0

Available online at

www.springerlink.com

\title{
ESTIMATION OF OSL TRAP PARAMETERS BY OPTICAL "CLEANING" - A CRITICAL STUDY
}

\author{
NATALIA KIJEK and ALICJA CHRUŚCIŃSKA \\ Institute of Physics, Faculty of Physics, Astronomy and Informatics, Nicolaus Copernicus University, Grudziądzka 5, 87-100 Toruń
}

Received 30 June 2013

Accepted 25 November 2013

\begin{abstract}
The so-called thermal "cleaning" applied in thermoluminescence analysis allows distinguishing TL signal originating from different traps of comparable thermal depths. Here, the detailed study on the suitability of a similar approach — which can be called optical "cleaning" — for the analysis of OSL process has been carried out by means of computer modelling. The optical "cleaning" is realized by short optical stimulation applied directly after irradiation. It turns out that this approach does not help to separate signals related to various traps having similar optical cross-section (OCS) values. For some sets of trap parameters, sufficiently good reconstruction of the trap optical cross-sections can be achieved by the analysis of the conventional OSL curve obtained directly after sample irradiation, while the OSL curve analysis after "cleaning" does not provide any additional data. For other cases the analysis of both curves with and without "cleaning" leads to optical crosssection values different from those assumed in the model, but just in such situations the optical "cleaning" can be helpful. The differences between the OSL components of the conventional curve and the one obtained after "cleaning" can be used as indicators of an incompatibility of the trap parameters obtained from the OSL analysis with their actual values.
\end{abstract}

Keywords: optically stimulated luminescence, optical cross sections, bleaching, simulations.

\section{INTRODUCTION}

In optically stimulated luminescence (OSL) dating of geological sediments, the discrimination between fast and slow components of the OSL signal is of great importance (Wintle, 2010; Weckwerth et al., 2011; Przegiętka and Chruścińska, 2013). Extracting the fast component, which can be easily bleached by sunlight during the sediment grain transport before the deposition, may allow obtaining data for sediments that were exposed to sunlight not long enough for the total zeroing of the OSL signal. On the other hand, using slower OSL components for date estimation may help to extend the time limit of

Corresponding author: N. Kijek

e-mail: natalia@fizyka.umk.pl dating when the slow component increases less rapidly with excitation dose than the fast one. At the present stage of the development of OSL measurement techniques, however, it is hard to separate the individual OSL components experimentally. The course of optical stimulation in both CW-OSL and LM-OSL measurement techniques causes the simultaneous decay of all OSL components (Chruścińska, 2007). However the fact that this decay is not equally efficient for the fast and slower components may suggest the way of component separation consisting in gradual optical "cleaning" of more and more slower components by repetition of several OSL measurements after one excitation (Hsieh et al., 2012; Kitis and Pagonis, 2008; Singarayer et al., 2000). The premise accepted here is that when one bleaches the major part of 
faster component, the next slower component can be observed more clearly as it is successfully realized by the so-called thermal "cleaning" in thermoluminescence analysis (Isik et al., 2012; Fasoli et al., 2010; Pagonis et al., 2009). Because literature lacks data concerning the influence of the optical "cleaning" on the subsequent OSL process, it is difficult to determine whether the analysis of the experimental results obtained as a result of such method can be useful. In this study, a detailed analysis of such approach by computer simulations of OSL process is presented. For explicit results a simple OSL model including two electron traps and one luminescence centre has been assumed. The set of differential equations for such a model has been solved for the 1) process of trap filling (excitation), 2) relaxation after excitation, 3) the first optical stimulation ("cleaning"), 4) relaxation after optical stimulation and 5) the second optical stimulation. The simulations have been carried out for different trap parameters (optical cross-section, concentration and retrapping parameter) and for different experimental conditions (excitation time and stimulation light intensity). The approach commonly used for OSL curve analysis, which consists of fitting of a sum of first order curves to an experimental curve has been applied to OSL curves obtained in simulations, as a result of both the first and the second optical stimulation. An outcome of such procedure is an optical cross-section spectrum that allows distinguishing the individual OSL components. On the basis of such analysis, first of all the need of optical "cleaning" has been evaluated.

One can reasonably assume that the second stimulation is useless when the fitting procedure applied for the results of first stimulation (step 3 of simulations) accurately reconstructs the OCSs postulated in the model. Moreover we show, that the interpretation of fitting results for the OSL curve measured after "cleaning" can lead to erroneous conclusions regarding the slow component. In the presented investigation special attention has been paid just to the quality of the reconstruction of the optical cross-section by the fitting analysis of OSL curve obtained during the second stimulation for the trap responsible for the slower component. It turns out that after the "cleaning" of the fast component the effects of trap coupling strongly influence the OSL process during the second stimulation. The analysis of OSL curve obtained at this stage can lead to OCS values deviating significantly from the real values postulated in the model. However, there are also some cases when the second stimulation after optical "cleaning" turns out to be useful.

\section{METHODS}

The model used for the OSL process simulations is composed of two electron traps and one recombination centre. Both electron traps are emptied during the optical stimulation. Five processes have been simulated numerically by solving differential equations in the following steps: trap filling during irradiation, relaxation after excitation, first optical stimulation bleaching the shallower trap, relaxation after optical stimulation and second optical stimulation. In this way one can investigate both the standard OSL curve and the "cleaning" effects. The kinetic equations solved at each step of simulations have the following form:

$$
\begin{aligned}
& \frac{d n_{i}}{d t}=-\gamma_{i} n_{i}+A_{i}\left(N_{i}-n_{i}\right) n_{c} ; \quad i=1,2 \\
& \frac{d m}{d t}=A_{m}(M-m) m_{v}-\beta m n_{c} \\
& \frac{d n_{c}}{d t}=R+\sum_{i=1}^{2}\left[\gamma_{i} n_{i}-A_{i}\left(N_{i}-n_{i}\right) n_{c}\right]-\beta m n_{c} \\
& \frac{d m_{v}}{d t}=R-A_{m}(M-m) m_{v}
\end{aligned}
$$

where $n_{\mathrm{i}}\left(\mathrm{cm}^{-3}\right)$ is the concentration of electrons in $i$-th trap, $N_{\mathrm{i}}\left(\mathrm{cm}^{-3}\right)$ means the concentration of $i$-th trap, $m$ $\left(\mathrm{cm}^{-3}\right)$ is the concentration of holes in recombination centres and $M\left(\mathrm{~cm}^{-3}\right)$ the concentration of these centres that was equal $10^{11} \mathrm{~cm}^{-3}$ in all the investigated cases, $n_{c}$ $\left(\mathrm{cm}^{-3}\right)$ and $m_{\mathrm{v}}\left(\mathrm{cm}^{-3}\right)$ are, respectively, concentrations of free electrons and holes, $A_{i}\left(\mathrm{~cm}^{3} \mathrm{~s}^{-1}\right)$ is the probability of electron trapping in $i$-th trap, $A_{\mathrm{m}}\left(\mathrm{cm}^{3} \mathrm{~s}^{-1}\right)$ is the probability of hole trapping in the recombination centre, $R\left(\mathrm{~cm}^{-3} \mathrm{~s}^{-}\right.$ ${ }^{1}$ ) is the intensity of the excitation irradiation producing the pairs of free electrons and holes during the trap filling process, $\beta_{\mathrm{i}}\left(\mathrm{cm}^{3} \mathrm{~s}^{-1}\right)$ is the probability of a free electron recombining with a hole trapped in the luminescence centre and it is equal $10^{-8} \mathrm{~cm}^{3} \mathrm{~s}^{-1}$ in all simulations. The value of probability of optical excitation of electron from the trap to the conduction band $\gamma_{\mathrm{i}}\left(\mathrm{s}^{-1}\right)$ is equal: $\gamma=\sigma_{\mathrm{i}} f$, where $\sigma_{\mathrm{i}}\left(\mathrm{cm}^{2}\right)$ is the OCS of $i$-th trap and $f\left(\mathrm{~cm}^{-2} \mathrm{~s}^{-1}\right)$ is the photon flux of stimulation light. The above equation set describes the trap filling during irradiation when $f=0$ and $R=10^{7} \mathrm{~cm}^{-3} \mathrm{~s}^{-1}$, relaxation when $f, R=0$ and optical stimulation when $f=10^{18} \mathrm{~cm}^{-2} \mathrm{~s}^{-1}$ and $R=0$. In all presented cases $\sigma_{1}$ is $10^{-19} \mathrm{~cm}^{2}$.

The model has been investigated mainly for such retrapping parameters for which each of the traps, if treated independently, would be emptied according to the firstorder kinetics $\left(A_{1}, A_{2} \leq \beta / 50\right)$. In fact, only for such parameter sets one can assume that fitting a sum of first order curves to a simulated OSL curve is reasonable. Some tests have been also carried out for parameters leading to non-first-order process (see section 3 - Effects related to the weak fulfillment of the first order kinetics). The results of these studies are mentioned because the optical "cleaning" turns out to be helpful in the recognition of this case.

The simulations have been performed with MATLAB differential equation solver ode23s, which is a tool suitable for stiff equation sets. In the procedure of fitting the 
sum of the first order OSL curves to the simulated OSL curves, performed to find the OSL signal components, MATLAB non-negative least squares procedure have been used. In this procedure, the OSL intensity is assumed to be:

$$
I(t)=\sum_{k} n_{o k} f \sigma_{k} \exp \left(-f \sigma_{k} t\right)
$$

where $\sigma_{\mathrm{k}}$ has its value in the range of $10^{-16}$ to $10^{-24} \mathrm{~cm}^{2}$.

It should be stressed that in all the obtained outcomes of fitting of a sum of first order components to the OSL curves has been very good. A result has been regarded correct only when the residual for every data point was lower than $1 \%$ of the OSL intensity. This method of verifying the reliability of the fitting results is much more restrictive then controlling the figure of merit which is equal:

$$
F O M=\frac{\sum_{i=1}^{N} 100\left[I_{i}-I_{i}^{d o p}\right]}{\sum_{i=1}^{N} I_{i}}
$$

where $I_{\mathrm{i}}$ denotes the value of intensity of OSL signal in $i$ th data point obtained from the simulations and $I_{\mathrm{i}}^{\text {dop }}$ is the intensity of OSL signal obtained from the fitting. The FOM in the presented fitting results is always much less than $1 \%$.

\section{RESULTS AND DISCUSSION}

\section{Effects of trap population}

The first fundamental observation that is common for all trap parameter sets is the dependence of the fitting results on the initial occupation of traps. The intensive study has been performed for different levels of trap occupation, from very low ones to the saturation level and it has been observed that the correct reconstruction of the OCS values and trap occupations can be obtained only after the excitation leading to very high, close to saturation level, population of electrons in traps. It means that the OSL parameter analysis performed for the natural signal is reasonable only when one is sure that the OSL signal is close to saturation or is saturated. Fig. 1 presents the effects obtained for traps having equal and very low retrapping coefficients (Fig. 1a) and for traps having different and larger retrapping coefficients (Fig. 1b). While for the traps of low $A_{1}$ and $A_{2}$ (cf. (1)-(4)) the OCS values are reconstructed properly (but their intensities much less acceptable), for larger $A_{1}$ and $A_{2}$ not only the component intensities are false but also the splitting of components can be so wide that an additional medium components can be recognized. This result should not be surprising because it is a direct consequence of the basic assumption of first-order kinetics that requires that $A_{\mathrm{i}}$ $\left(N_{\mathrm{i}}-n_{\mathrm{i}}\right)<<\beta m$. Weakly populated traps make the factor
$\left(N_{\mathrm{i}}-n_{\mathrm{i}}\right)$ higher which weakens the above condition, so the fitting of the sum of first-order curves cannot give proper results, even when the fitting results are correct from the formal point of view.

Here it should be noted that in practice, when investigating the OSL signal, one does not know the values of retrapping coefficients of traps active in the OSL process. Therefore in order to avoid the worst case scenario one should use such experimental conditions that assure the best possible results. In other words, high levels of trap filling should be achieved.

The problem of trap occupation is closely related to the quality of OCS spectrum determination from the OSL curve resulting from the stimulation after optical cleaning. Accordingly, the results of the second stimulation obviously depend on the time of "cleaning" because it changes the occupation of traps. Investigations performed for different times of optical "cleaning" show that in the cases when very good fitting results are obtained in the first stimulation (see next subsections), the time of "cleaning" has no special effect on the OCS values obtained in the second stimulation. There are discrepancies in the component intensities obtained from this procedure and the intensities actually reached after the simulation of "cleaning" process. The more radical changes of OCS spectra with the time of "cleaning" are observed for trap parameters, which make the results of analysis of the first stimulation problematic. Here, short "cleaning" times lead to the significant splitting of the faster component. Longer times, that allow significant depopulation not only of the shallower trap but also of the deeper one, generate an additional intensive component that is clearly slower than the slow component assumed in the model. In all presented results the shallower trap has the same value of OCS $\left(10^{-19} \mathrm{~cm}^{2}\right)$, so for the purpose of this work, the most convenient "cleaning" time was found to be equal to $40 \mathrm{~s}$. This time of "cleaning" sufficiently reduces the concentration of electrons in this trap, so the results of the mentioned splitting are not confusing. Simultaneously, it does not empty the deeper trap significantly, even when its OCS is only slightly smaller than the OCS of shallower trap.

\section{Possibility of separating the signal from different traps}

The properly performed analysis of the OSL curve gives good results in the case of traps having very close OCS's only for the first optical stimulation and for sufficiently low retrapping coefficients (Fig. 1a). As can be seen in Fig. 2c, for the same model parameters, but higher retrapping coefficients, weakening the assumptions of first order kinetics, two kinds of traps are recognized as a single one of double population. The results of the estimation of OSL components for the second stimulation repeated after the partial emptying of traps ("cleaning") are inconsistent with the real state of the system even though the retrapping coefficients of both traps are very 


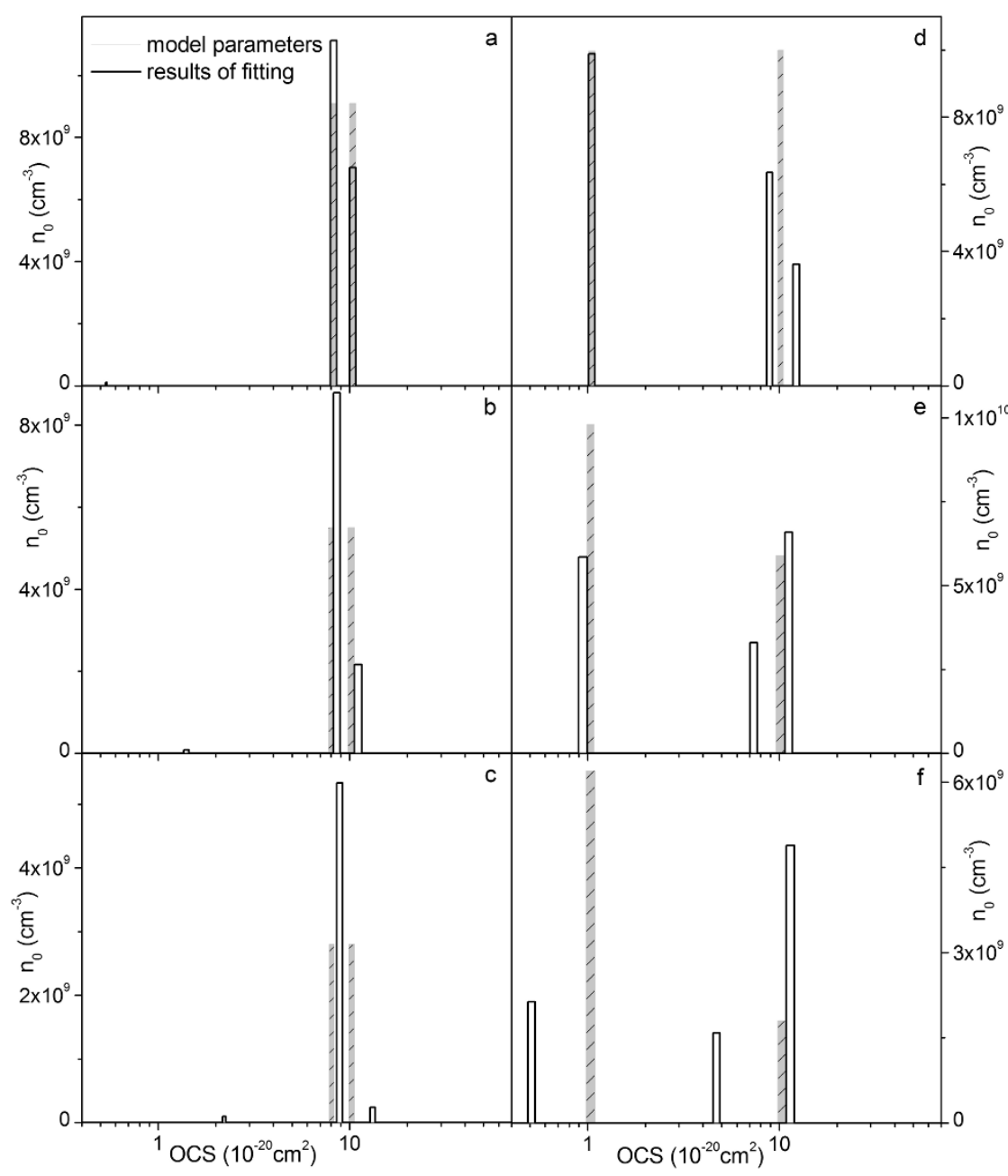

Fig. 1. Results of fitting the first order curves to simulated OSL curves - the effect of initial trap occupation on the reconstruction of OSC spectra by fitting applied to OSL curve obtained in the first stimulation after irradiation. Two different cases are presented. The first one for model parameters that fulfill the assumptions of first-order kinetics very strictly and for initial trap occupation at $91 \%$ (a), $55 \%$ (b) and $28 \%$ (c) of the saturation level. The model parameters used in simulations for this case are: $A_{1}=A_{2}=10^{-11} \mathrm{~cm}^{3} \mathrm{~s}^{-1}, \quad N_{1}=N_{2}=10^{10} \mathrm{~cm}^{-3}$, $\sigma_{2}=8 \times 10^{-20} \mathrm{~cm}^{2}$. The second case illustrates

. the worse fitting results obtained for higher E retrapping coefficients and for initial trap $\approx^{\circ}$ occupation at $99 \%$ of the saturation level (a), at $99 \%$ for shallower trap and at $58 \%$ for the deeper trap (b) at $63 \%$ for the shallower trap and at $18 \%$ for the deeper trap (c). The trap parameters used in this case are: $A_{1}=5 \times 10^{-10} \mathrm{~cm}^{3} \mathrm{~s}^{-1}, \quad \mathrm{~A}_{2}=10^{-10} \mathrm{~cm}^{3} \mathrm{~s}^{-1}$, $\mathrm{N}_{1}=\mathrm{N}_{2}=10^{10} \mathrm{~cm}^{-3}, \quad \sigma_{2}=1 \times 10^{-20} \mathrm{~cm}^{2}$. The higher occupation of traps enables the reconstruction of OCS values that reflects the values assumed in the model (even for the relatively high retrapping coefficient that weakens the first order kinetics assumption (d)) whereas the low electron concentration in traps $(e, f)$ leads to the appearance of an additional OSL component that is not related to any trap assumed in the model.

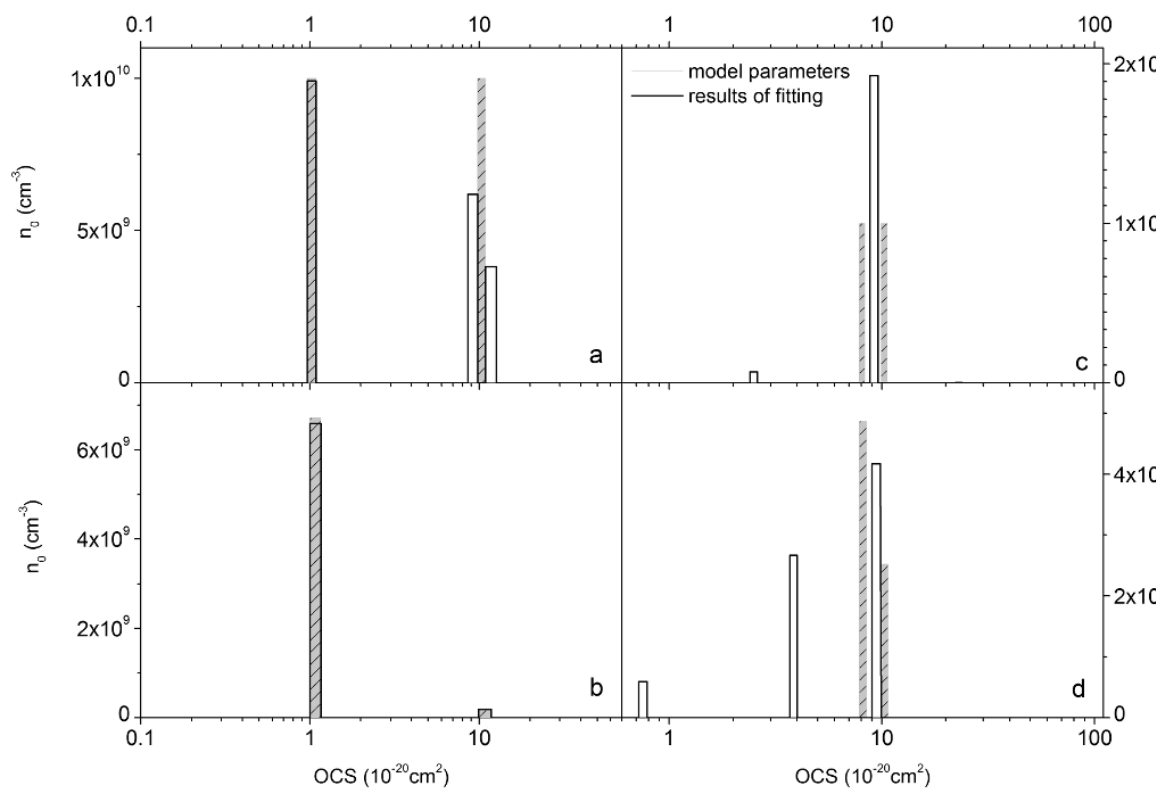

Fig. 2. Results of the first order curve fitting to the OSL curves: upper - simulated for the first stimulation (performed directly after irradiation) and lower - for the second stimulation (performed after a short optical stimulation - "cleaning") - the capability of discriminating different kinds of traps by OSL measurement. $(a, b)$ - Illustration of very good results of the fitting obtained for traps having significantly different OCSs: the parameters used for simulations: (a): $\mathrm{A}_{1}=\mathrm{A}_{2}=10^{-10} \mathrm{~cm}^{3} \mathrm{~s}^{-1}, \quad \mathrm{~N}_{1}=\mathrm{N}_{2}=10^{10} \mathrm{~cm}^{-3}$, $\sigma_{2}=10^{-20} \mathrm{~cm}^{2}$, texc $=3 \times 10^{6} \mathrm{~s}$ and $(b)$ : like in (a) but after $40 \mathrm{~s}$ of optical "cleaning". (c, d) - the case showing the effects observed for very close OCS values; the parameters used $2 \times 10^{8}$ for simulations: (c): $A_{1}=A_{2}=10^{-10} \mathrm{~cm}^{3} \mathrm{~s}^{-1}$, $\mathrm{N}_{1}=\mathrm{N}_{2}=10^{10} \mathrm{~cm}^{-3}, \quad \sigma_{2}=8 \times 10^{-20} \mathrm{~cm}^{2}$, $t_{\text {exc }}=3 \times 10^{6} s$ and (d): like in (c) after $40 \mathrm{~s}$ of "cleaning". 
small (Fig. 1b). In the case when the first order kinetics assumption is slightly weakened, the results of OSL curve decomposition are very confusing: instead of two close OCSs one can observe three different components whose intensities cannot be neglected (Fig. 2d). This is a simple consequence of the above-mentioned rule concerning the proper trap filling for the OSL analysis. The traps of very close OCS are depopulated to a very similar degree and both traps are considerably emptied during the "cleaning" which is meant to depopulate only the shallower one. For sufficiently distant OCSs good results can be obtained for both stimulations for a certain range of trap parameters (see the next sections), however, the question arises what is the purpose of measuring the second OSL curve after "cleaning" when analysis of the first curve gives satisfactory results.

\section{The low concentration of the shallower trap}

Good fitting results for both the first and the second stimulation are obtained for cases when the concentration of the deeper trap $N_{2}$ is not smaller than the concentration of the shallower trap $N_{1}\left(N_{1} \leq N_{2}\right)$ and when the retrapping coefficients of traps meet one of the following conditions:

- $A_{1} \leq A_{2} \leq \beta / 50$ (see the example in Fig. 3a and b);

- $\quad A_{1}>A_{2}$ and $A_{1}, A_{2} \leq \beta / 100$ (see the example in Fig. 3c and $\mathbf{d}$ ).

The examples presented in Fig. 3 for $N_{1}=N_{2}$ are chosen for a radical situation when the analysis outcomes can give less satisfying results. As can be seen, they are still acceptable and the tendency is such that the larger the difference $N_{1}-N_{2}$, the better the reconstruction of OCS spectrum by the fitting procedure. As mentioned above, the general condition here is the sufficiently large difference between the OCS's of both traps. Obviously, in these cases there is no need to apply the optical cleaning. The experimental evidence of the above combination of trap parameters, in the results of first stimulation, are the clearly distinguishable OSL components where the slower one is at least as strong as the fast one, or stronger, which is even more propitious.

When the above conditions are not fulfilled, which means that $A_{1}>A_{2}$ and $A_{1}, A_{2}>\beta / 100$, the OCS spectra obtained for the first stimulation do not agree with the real values of OCS assumed in the model. The disadvantageous effect observed in these cases is the splitting of the OSL component into two (or even three for higher $A_{1} / A_{2}$ ratios) clearly different components (Fig. 4a). Fig. 5 sums up of the detailed investigation carried out for these problematic cases. For each case all the parameters of the model are kept constant except the retrapping parameter for the shallower trap $A_{1}$. The changes of the OCS values obtained by fitting are shown for the increasing $A_{1} / A_{2}$ ratio and for concentration relation $N_{1}=N_{2}$ (for lower $N_{1}$ concentrations theses effects are similar). Close OCS values of traps, as it has been mentioned earlier, may lead to very confusing results (Fig. 5a). The main outcome is the increasing (with $A_{1} / A_{2}$ ratio) number of the slower OSL components and a continuous shift of the OCSs to higher values.

As it can be seen, for the significant difference between the OCSs of traps (Fig. 5b), the main outcome is the splitting of the both components into two weaker ones and, for higher values of the $A_{1} / A_{2}$ ratio, the appearance of an additional medium component. In the described cases the second stimulation after optical "cleaning" can help to recognize which OSL components are connected

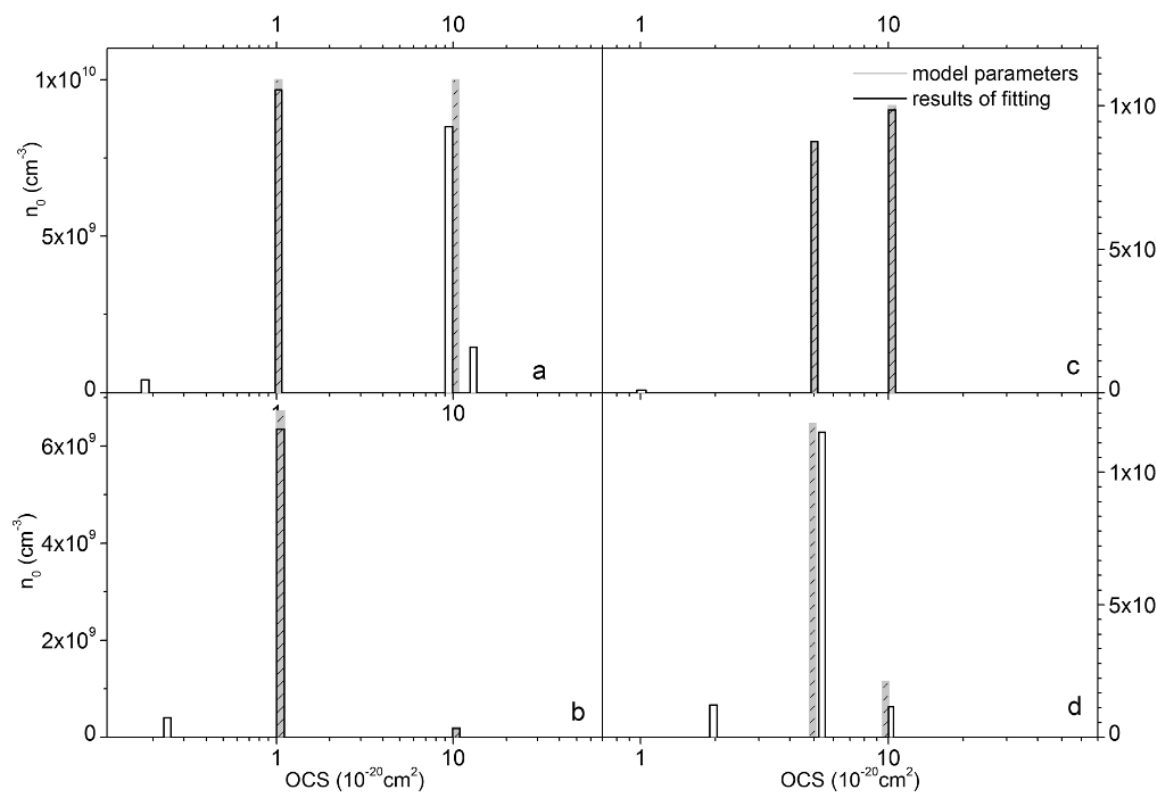

Fig. 3. OCS spectra that acceptably reconstruct the model assumptions obtained by $\times 10^{10}$ fitting procedure for the first (upper) stimulation and second (lower) stimulation after optical "cleaning" (40 s) for the case when $\mathrm{N}_{1} \leq \mathrm{N}_{2}$ and for the combinations of trap $10^{9}$ parameters that assure the minimal effects of trap coupling and hence the conformity of the OSL process with the first-order kinetics. (a, b) - the borderline case for $A_{1}=A_{2}=\beta / 50$ (for lower values of $A_{1}=A_{2}$ fitting results are definitely better) and $(c, d)$ - the case of close OCS values which for higher $\mathrm{A}_{1}$ and $\mathrm{A}_{2}$ leads to poor reconstruction of OCS. The relations $A_{1}=\beta / 200$ and $A_{2}=\beta / 1000$ guarantee first-order OSL kinetics for each trap treated independently. The parameters used for the simulations are: (a) and (b) $\mathrm{A}_{1}=\mathrm{A}_{2}=2 \times 10^{-10} \mathrm{~cm}^{3} \mathrm{~s}^{-1}, \mathrm{~N}_{1}=\mathrm{N}_{2}=10^{10} \mathrm{~cm}^{-3}$, $\sigma_{2}=10 \times 10^{-20} \mathrm{~cm}^{2} ; \quad$ (c) and (d) $\mathrm{A}_{1}=5 \times 10^{-11} \mathrm{~cm}^{3} \mathrm{~s}^{-1}, \quad \mathrm{~A}_{2}=10^{-11} \mathrm{~cm}^{3} \mathrm{~s}^{-1}$, $\mathrm{N}_{1}=\mathrm{N}_{2}=10^{10} \mathrm{~cm}^{-3}, \quad \sigma_{2}=5 \times 10^{-20} \mathrm{~cm}^{2}$, $t_{e x c}=3 \times 10^{6} \mathrm{~s}$ for both cases. 
with actual traps existing in the material (Fig. 4b). If after short optical "cleaning" a few faster components disappear, one can conclude that they all are related to one kind of traps. Moreover the lower OCS values, which are obtained from the analysis of first stimulation OSL curve approximate the OCS of this trap more accurately (Fig. 4a, Fig. 5b).

\section{The case of high concentration of the shallower trap}

There are arrangements of trap parameters that always result (already in the first stimulation) in OCS spectra that do not agree with the real values of OCS assumed in the model. It is worth recalling that all tests presented here are performed for the retrapping parameter values that guarantee the first-order kinetics for each trap treated independently. Nevertheless in the case when $N_{1}>N_{2}$ the characteristic outcome is the appearance of an additional artificial OSL component having intermediate OCS value between those of real traps (Fig. 4c). The reason of these effects is the enhancement of retrapping in the numerous empty shallower traps at the moment when the deeper traps are effectively depopulated. This is again the case when the second stimulation after optical "cleaning" can help to decide which OSL components are related to real traps (Fig. 4c and $\mathbf{d}$ ). The deeper trap of high concentration can be easily recognized in the second OSL stimulation curve, the faster component vanishes, whereas the
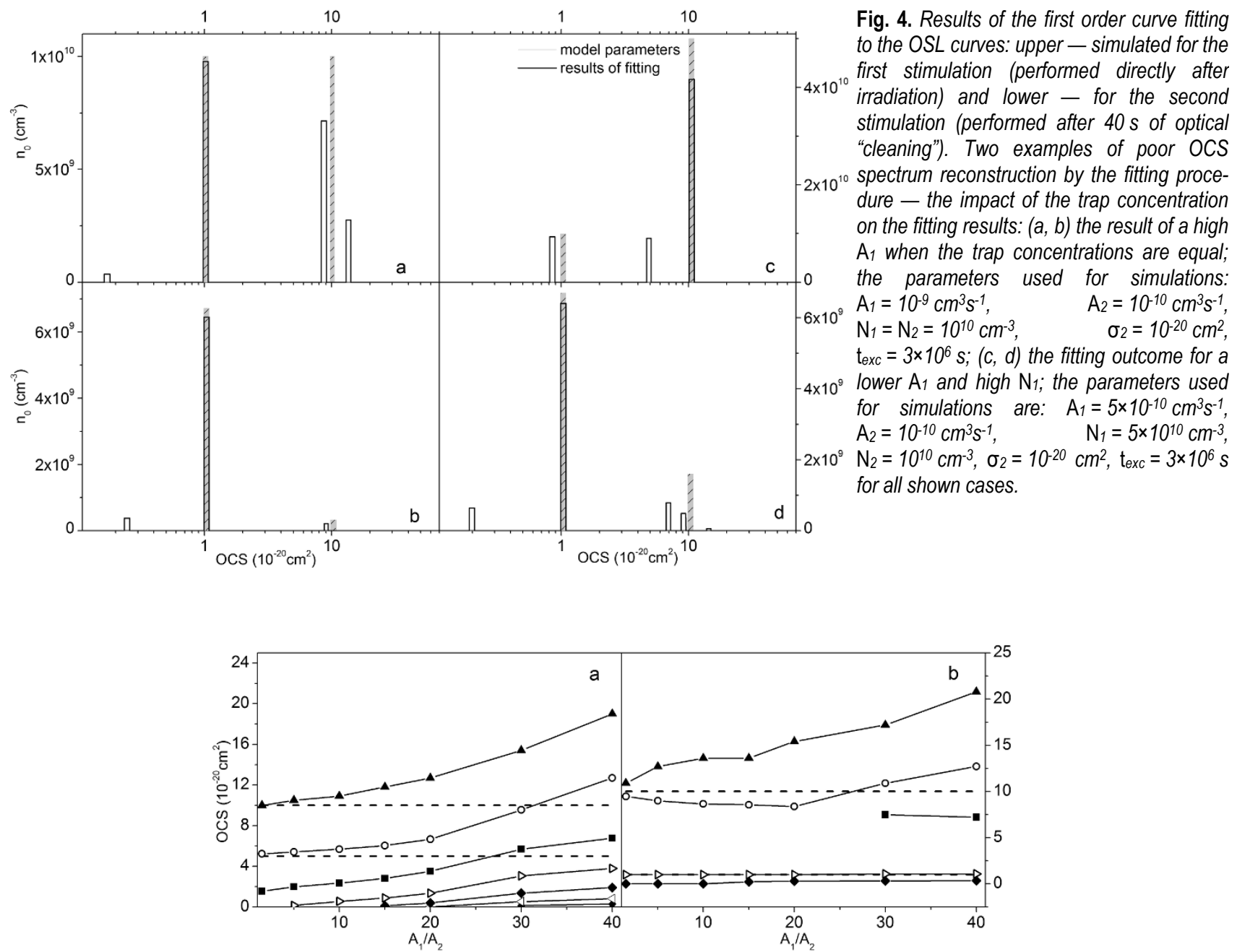

Fig. 5. Conclusions of the study performed for low concentrations of shallower trap and different values of the $\mathrm{A}_{1} / \mathrm{A}_{2}$ ratio. Presented are the examples for $\mathrm{N}_{1}=\mathrm{N}_{2}=10^{10} \mathrm{~cm}^{-3}$. In all cases when $\mathrm{N}_{1}<\mathrm{N}_{2}$ the effects are less disadvantageous. Figures show the OCS values obtained from the fitting procedure used for the first stimulation. $\mathrm{A}_{2}$ is constant and equal $10^{-10} \mathrm{~cm}^{3} \mathrm{~s}^{-1}$. Simulations were performed for subsequent $\mathrm{A}_{1}$ values $\left(\mathrm{cm}^{3} \mathrm{~s}^{-1}\right)$ : $1.5 \times 10^{-10}, 5 \times 10^{-10}, 10^{-9}, 1.5 \times 10^{-9}, 2 \times 10^{-9}, 3 \times 10^{-9}, 4 \times 10^{-9}$. Lines connecting results related to an individual OSL component are used in order to increase the clarity of the figure. (a) - the results for the close OCS values of traps, $\sigma_{2}=5 \times 10^{-20} \mathrm{~cm}^{2}$, some new OSL components turn up with the increasing $\mathrm{A}_{1} / \mathrm{A}_{2}$ ratio (b) - the results for well-separated traps, $\sigma_{2}=10^{-20} \mathrm{~cm}^{2}$ the number of components is more stable but larger than it is assumed in the model and the OSC value of shallower trap changes significantly; texc $=3 \times 10^{6} \mathrm{~s}$ for both cases. Dashed lines represent the OCS values assumed in the simulations. 
intermediate component decays and its OCS value increases (Fig. 4d). Such increasing OCS with longer time of optical "cleaning" should be considered as an indication of questionable reality of the OSL medium component.

\section{Effects related to the weak fulfillment of the first or- der kinetics}

For retrapping coefficients which are not small enough in comparison with the radiation recombination coefficient of luminescence centre, so that the assumptions of first-order kinetics are not fulfilled, one can observe some effects that can help to recognize this case. To this end, the comparison of fitting results for the first stimulation with these obtained after "cleaning" is useful. It is easy to notice that the OCS values in both spectra are different. Slower components become faster and new slow components turn up in OCS spectra for second stimulation. Such effects should call for a careful evaluation of fitting results (Fig. 6). In fact, for fundamental reasons, the fitting of the first order curves in this case cannot be expected to give correct results.

\section{CONCLUSIONS}

The analysis of simulated OSL curves shows that good fitting (with a very small FOM) of first-order kinetics curves to the simulated curve can be obtained even for trap parameters that are rather far from the values that meet the first-order kinetics assumption. Consequently the results of such approach have to be treated with great caution. First of all, fitting can be applied only to samples irradiated in the laboratory for a sufficiently long time, ensuring high - i.e. close to saturation - filling of traps. Such analysis can be performed only for old naturally excited samples after a control of the level of trap occupation. In general, because of the correlation with conditions $A_{1}<A_{2}$ and $N_{1}<N_{2}$, the cases when the slower component has higher intensity are favorable for good reconstruction of OCS spectra by fitting with the first order curves. In such cases the analysis applied to the optically "cleaned" OSL signal does not give any new information.

In general, there is a rather narrow range of cases for which it makes sense to apply the optical "cleaning". This approach does not help to separate signals originating from traps having close OCS values, which is an advantage of thermal "cleaning" in TL analysis. It also gives mostly weak results in the cases when the analysis of the curve from first optical stimulation after irradiation is not satisfactory. However optical "cleaning" can be helpful in the cases when some OSL components (mainly medium and slow ones) appearing in the OCS spectrum for first stimulation are questionable and this is usually the case when the fast component is most intensive. The false medium component disappears from the OCS spectrum for second stimulation and the artificial slow components have higher OCS values.

\section{ACKNOWLEDGEMENTS}

This work has been partially financed by the grant of the Polish National Center for Research and Development No. PBS1/A9/4/2012 and by the grant No. 1137-F obtained from Nicolaus Copernicus University.

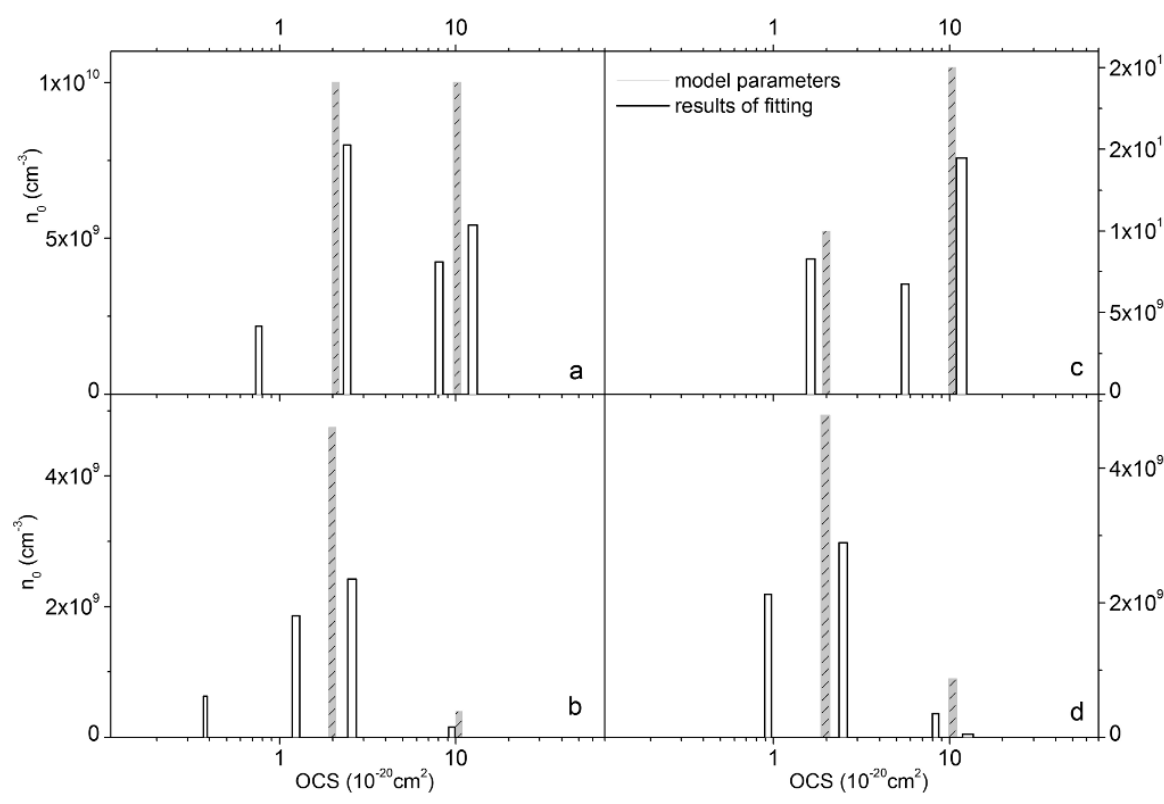

Fig. 6. Effects of applying the first-order curve fitting in the cases when the first-order kinetics assumptions are not fulfilled. Results shown for the first stimulation (upper) and second stimulation performed after $40 \mathrm{~s}$ of optical "cleaning" (lower). (a, b) - the case of high values of $A_{1}$ and $A_{2}$ and equal trap concentrations: $\quad A_{1}=A_{2}=10^{-9} \mathrm{~cm}^{3} \mathrm{~s}^{-1}$, $\mathrm{N}_{1}=\mathrm{N}_{2}=10^{10} \mathrm{~cm}^{-3} ;(c, d)$ - the characteristic example for high values of $\mathrm{A}_{1}$ and $\mathrm{A}_{2}$ and higher concentration of the shallower trap: $\mathrm{A}_{1}=\mathrm{A}_{2}=10^{-9} \mathrm{~cm}^{3} \mathrm{~s}^{-1}, \quad \mathrm{~N}_{1}=2 \times 10^{10} \mathrm{~cm}^{-3}$, $\mathrm{N}_{2}=10^{10} \mathrm{~cm}^{-3}$. Efficient depopulation of medium component during short optical "cleaning" together with an increase of its OCS value and the emerging some new slow components ( $b$ and $d$ ) in the OCS spectrum for the second stimulation are signs that caution is necessary in interpreting the results of both the first and second stimulation. In both cases $\sigma_{2}=2 \times 10^{-20} \mathrm{~cm}^{2}$ and $t_{e x c}=3 \times 10^{6} \mathrm{~s}$. 


\section{REFERENCES}

Chruścińska A, 2007. Complex OSL signal and a trap independence assumption. Radiation Measurements 42(4-5): 727-730, DOI 10.1016/j.radmeas.2007.02.026.

Fasoli M, Vedda A, Moretti F, Chenus AC, Veronese I, Cantone MC, Nik1 M, Yoshikawa A and Novoselov A, 2010. Effect of Eu and $\mathrm{Pb}$ doping on the dosimetric properties of LiCAF. Radiation Measurements 45(3-6): $\quad 556-558, \quad$ DOI 10.1016/j.radmeas.2010.01.026.

Hsieh C-W, Tiu C-M and Li J-W, 2012. Non-linear dose study of commercial $\mathrm{Al}_{2} \mathrm{O}_{3}: \mathrm{C}$ optically stimulated luminescence dosimeter with partial optical bleaching. CIC Express Letters, Part B: Applications 3: 615-620.Isik M, Bulur E and Gasanly NM, 2012. Lowtemperature thermoluminescence in layered structured $\mathrm{Ga}_{0.75} \mathrm{In}_{0.25}$ Se single crystals. Journal of Alloys and Compounds 545: 153-156, DOI 10.1016/j.jallcom.2012.08.015.

Kitis G and Pagonis V, 2008. Computerized curve deconvolution analy sis for LM-OSL. Radiation Measurements 43(2-6): 737-741, DOI 10.1016/j.radmeas.2007.12.055.

Pagonis V, Mian S, Mellinger R and Chapman K, 2009. Thermoluminescence kinetic study of binary lead-silicate glasses. Journal of Luminescence $\quad 129(5): \quad 570-577$,

DOI 10.1016/j.jlumin.2008.12.016.

Przegiętka KR and Chruścińska A, 2013. Analysis of optical bleaching of OSL signal in sediment quartz. Radiation Measurements 56: 257-261, DOI 10.1016/j.radmeas.2013.02.009.

Singarayer JS, Bailey RM and Rhodes EJ, 2000. Potential of the slow component of quartz OSL for age determination of sedimentary samples. Radiation Measurements 32(5-6): 873-880, DOI 10.1016/S1350-4487(00)00074-3.

Weckwerth P, Przegiętka KR, Chruścińska A, Woronko B and Oczkowski HL, 2011. Age and sedimentological features of fluvial series in the Torun Basin and the Drwęca Valley (Poland). Geochronometria 38(4): 397-412, DOI 10.2478/s13386-011-0038-1.

Wintle AG, 2010. Future directions of luminescence dating of quartz. Geochronometria 37: 1-7, DOI 10.2478/v10003-010-0023-3. 